\title{
Primitive Points on a Modular Hyperbola
}

by

\author{
Igor E. SHPARLINSKI \\ Presented by Andrzej SCHINZEL
}

Summary. For positive integers $m, U$ and $V$, we obtain an asymptotic formula for the number of integer points $(u, v) \in[1, U] \times[1, V]$ which belong to the modular hyperbola $u v \equiv 1(\bmod m)$ and also have $\operatorname{gcd}(u, v)=1$, which are also known as primitive points. Such points have a nice geometric interpretation as points on the modular hyperbola which are "visible" from the origin.

1. Introduction. For a positive integer $m$ we consider the modular hyperbola

$$
\mathcal{H}_{m}=\{(u, v): u v \equiv 1(\bmod m), 1 \leq u, v<m\} .
$$

Various properties of the points $(u, v) \in \mathcal{H}_{m}$ have been considered in the literature. For example,

- the question about the joint distribution of parity of $u$ and $v$ is known as the Lehmer problem and has attracted a lot of attention (see [27]$[29])$;

- the distribution of the distances $|u-v|$ for $(u, v) \in \mathcal{H}_{m}$ has been addressed in the literature as well (see $[5,14,30])$;

- some geometric properties of the convex hull of $\mathcal{H}_{m}$ have been studied in [15].

Here we consider an apparently new question of estimating the number of points $(u, v) \in \mathcal{H}_{m}$ with $\operatorname{gcd}(u, v)=1$ which belong to a given box $(u, v) \in$ $[1, U] \times[1, V]$. These points have an attractive geometric interpretation as points on $\mathcal{H}_{m}$ which are "visible" from the origin (see $[2,12,18,26]$ and references therein for several other aspects of distribution of "visible" points in various regions).

2000 Mathematics Subject Classification: 11A07, 11K38, 11L40.

Key words and phrases: modular hyperbola, primitive point.

This work was supported in part by ARC grant DP0556431. 
More precisely, for positive real numbers $U$ and $V$ we consider the set

$$
\mathcal{H}_{m}(U, V)=\left\{(u, v) \in \mathcal{H}_{m}: 1 \leq u \leq U, 1 \leq v \leq V\right\}
$$

and we define

$$
N_{m}(U, V)=\sum_{\substack{(u, v) \in \mathcal{H}_{m}(U, V) \\ \operatorname{gcd}(u, v)=1}} 1 .
$$

We obtain an asymptotic formula for $N_{m}(U, V)$ which is nontrivial whenever

$$
U V \geq m^{3 / 2+\varepsilon}
$$

for any fixed $\varepsilon>0$ and sufficiently large $m$.

We recall that the notations $U \ll V$ and $U=O(V)$ are both equivalent to the statement that $|U| \leq c V$ with some constant $c>0$. Throughout the paper, $o(1)$ denotes a quantity which tends to zero as $m \rightarrow \infty$.

2. Preparation. We need the following bound on the distribution of inverses of squares in residue rings which could be of independent interest.

For an integer $d$ with $\operatorname{gcd}(d, m)=1$, we use $\bar{d}$ to denote the modular inverse of $d$ modulo $m$, that is, $d \bar{d} \equiv 1(\bmod m), 1 \leq \bar{d}<m$.

For a real $R$ and integers $K$ and $L$ with $1 \leq K, R<m$ we denote by $T_{m}(R ; K, L)$ the number of integers $d \in[L, L+K-1]$ with $\operatorname{gcd}(d, m)=1$ and such that $\overline{d^{2}} \equiv r(\bmod m)$ for some integer $r$ with $1 \leq r \leq R$.

Lemma 1. For any real $R$ and integers $K$ and $L$ with $1 \leq K, R<m$, we have

$$
T_{m}(R ; K, L)=\frac{R}{m} \sum_{\substack{d=L \\ \operatorname{gcd}(d, m)=1}}^{L+K-1} 1+O\left(m^{1 / 2+o(1)}\right) .
$$

Proof. The proof uses very standard arguments so we give only the main ingredients.

Our basic ingredient is the following bound on complete exponential sums:

$$
\max _{b=1, \ldots, m}\left|\sum_{\substack{d=1 \\ \operatorname{gcd}(d, m)=1}}^{m} \exp \left(2 \pi i \frac{a \overline{d^{2}}+b d}{m}\right)\right| \leq(m \operatorname{gcd}(a, m))^{1 / 2+o(1)},
$$

which holds for any integer $a$ and is a very special case of the more general bound of [20] for exponential sums with monomials. Now, using the standard reduction between complete and incomplete sums (see [13, Section 12.2]), we obtain

$$
\left|\sum_{\substack{d=L \\ \operatorname{gcd}(d, m)=1}}^{L+K-1} \exp \left(2 \pi i \frac{a \overline{d^{2}}}{m}\right)\right| \leq(m \operatorname{gcd}(a, m))^{1 / 2+o(1)} .
$$


Combining this with the Erdős-Turán inequality (see [17, Corollary 1.1, Chapter 1]), after simple calculations we obtain the desired result.

We also remark that the Weil and Salié bounds of complete Kloosterman sums together imply that

$$
\left|\sum_{\substack{u=1 \\ \operatorname{gcd}(u, m)=1}}^{m} \exp \left(2 \pi i \frac{a u+b \bar{u}}{m}\right)\right| \leq(m \operatorname{gcd}(a, m))^{1 / 2+o(1)}
$$

(see [13, Corollary 11.12]). Now, the above mentioned reduction between complete and incomplete sums (see [13, Section 12.2]) leads to the following well known bound on incomplete Kloosterman sums.

Lemma 2. For any integer a and real $Z$ with $1 \leq Z \leq m$, we have

$$
\sum_{\substack{(u, v) \in \mathcal{H}_{m} \\ 1 \leq u \leq Z}} \exp \left(2 \pi i \frac{a v}{m}\right) \leq(m \operatorname{gcd}(a, m))^{1 / 2+o(1)} .
$$

3. Main result. As usual, $\varphi(m)$ denotes the Euler function.

THEOREM 3. For all integers $m$ and real $U, V$ with $1 \leq U, V<m$, we have

$$
N_{m}(U, V)=\frac{6}{\pi^{2}} \cdot \frac{U V}{m} \prod_{p \mid m}\left(1+\frac{1}{p}\right)^{-1}+O\left(U^{1 / 2} V^{1 / 2} m^{-1 / 4+o(1)}\right),
$$

where the product is taken over all prime numbers $p \mid m$.

Proof. For an integer $d$, we let

$$
M_{m}(d ; U, V)=\sum_{\substack{(u, v) \in \mathcal{H}_{m}(U, V) \\ d \mid \operatorname{gcd}(u, v)}} 1
$$

be the number of pairs $(u, v) \in \mathcal{H}_{m}(U, V)$ with $d \mid \operatorname{gcd}(u, v)$.

Let $\mu(d)$ denote the Möbius function. We recall that $\mu(1)=1, \mu(d)=0$ if $d \geq 2$ is not square-free and $\mu(d)=(-1)^{\omega(d)}$ otherwise, where $\omega(d)$ is the number of distinct prime divisors of $d$. By the inclusion-exclusion principle, we write

$$
N_{m}(U, V)=\sum_{d=1}^{\infty} \mu(d) M_{m}(d ; U, V)
$$

Clearly

$$
M_{m}(d ; U, V)=0
$$

if $\operatorname{gcd}(d, m)>1$ or $d>m$. 
For $\operatorname{gcd}(d, m)=1$, writing

$$
u=d s \quad \text { and } \quad v=d t,
$$

we have

$$
M_{m}(d ; U, V)=\#\left\{(s, t): s t \equiv \overline{d^{2}}(\bmod m), 1 \leq s \leq U / d, 1 \leq t \leq V / d\right\}
$$

where as before, $\bar{d}$ denotes the modular inverse of $d$ modulo $m$.

Lemma 2, combined with the Erdős-Turán inequality (see [17, Corollary 1.1, Chapter 1]), immediately implies that

$$
M_{m}(d ; U, V)=\frac{U V \varphi(m)}{d^{2} m^{2}}+O\left(m^{1 / 2+o(1)}\right)
$$

(see, for example, [2, Lemma 1.7]; similar results are also obtained in $[14,29])$.

We also note that for each $d$, the product $r=s t \leq U V / d^{2}$, where $s$ and $t$ are given by (4), belongs to a fixed residue class modulo $m$ and thus can take at most $U V / d^{2} m+1$ possible values. Denoting by $\tau(k)$ the number of positive integer divisors of $k \geq 1$, we see that for each fixed $r \leq U V / d^{2} \leq U V \leq m^{2}$, there are $\tau(r)=m^{o(1)}$ pairs $(s, t)$ of integers $s$ and $t$ with $r=s t$ (see [24, Section I.5.2]). Therefore, we also have

$$
M_{m}(d ; U, V) \leq\left(\frac{U V}{d^{2} m}+1\right) m^{o(1)} .
$$

Finally, we note that for any integer $\Delta \geq \sqrt{U V / m}$ we have

$$
\sum_{2 \Delta>d \geq \Delta} M_{m}(d ; U, V) \leq T_{m}\left(U V / \Delta^{2} ; \Delta, \Delta\right) m^{o(1)}
$$

since $\overline{d^{2}} \equiv r(\bmod m)$ where, as before, $r=s t \leq U V / d^{2} \leq U V / \Delta^{2} \leq m$ (thus for every $d$ the value of $r$ is uniquely defined and for every $r$ there are at most $\tau(r)=m^{o(1)}$ possible pairs $\left.(s, t)\right)$. Therefore,

$$
\begin{aligned}
\sum_{m \geq d \geq \Delta} M_{m}(d ; U, V) & \leq \sum_{\nu=0}^{\lceil 2 \log m\rceil} \sum_{2^{\nu+1}} M_{\Delta>d \geq 2^{\nu} \Delta}(d ; U, V) \\
& \leq \sum_{\nu=0}^{\lceil 2 \log m\rceil} T_{m}\left(U V /\left(2^{\nu} \Delta\right)^{2} ; 2^{\nu} \Delta, 2^{\nu} \Delta\right) m^{o(1)} .
\end{aligned}
$$

Hence, by Lemma 1 we obtain

$$
\begin{aligned}
\sum_{m \geq d \geq \Delta} M_{m}(d ; U, V) & \leq \sum_{\nu=0}^{\lceil 2 \log m\rceil}\left(\frac{2^{\nu} \Delta U V}{\left(2^{\nu} \Delta\right)^{2} m^{1+o(1)}}+m^{1 / 2+o(1)}\right) \\
& \ll \frac{U V}{\Delta m^{1+o(1)}}+m^{1 / 2+o(1)} .
\end{aligned}
$$


Therefore, for arbitrary integers $\Delta>\delta>1$, using the asymptotic formula (5) for $d \leq \delta$, the bound (6) for $\delta<d \leq \Delta$, and the bound (7) for $d \geq \Delta$, we derive from (2) and (3) that

$$
N_{m}(U, V)=\frac{U V \varphi(m)}{m^{2}} \sum_{\substack{1 \leq d \leq \delta \\ \operatorname{gcd}(d, m)=1}} \frac{\mu(d)}{d^{2}}+E,
$$

where

$$
\begin{aligned}
E & \ll \delta m^{1 / 2+o(1)}+\sum_{\delta \leq d \leq \Delta}\left(\frac{U V}{d^{2} m}+1\right) m^{o(1)}+U^{1 / 2} V^{1 / 2} \Delta^{-1} m^{o(1)} \\
& \ll \delta m^{1 / 2+o(1)}+U V \delta^{-1} m^{-1}+\Delta m^{o(1)}+U V \Delta^{-1} m^{-1} .
\end{aligned}
$$

We also have

$$
\sum_{\substack{1 \leq d \leq \delta \\ \operatorname{gcd}(d, m)=1}} \frac{\mu(d)}{d^{2}}=\sum_{\substack{d \geq 1 \\ \operatorname{gcd}(d, m)=1}} \frac{\mu(d)}{d^{2}}+O\left(\delta^{-1}\right)=\prod_{p \nmid m}\left(1-\frac{1}{p^{2}}\right)+O\left(\delta^{-1}\right),
$$

where the product is taken over all prime numbers $p \nmid m$. Recalling that

$$
\prod_{p}\left(1-\frac{1}{p^{2}}\right)=\sum_{d \geq 1} \frac{\mu(d)}{d^{2}}=\zeta(2)^{-1}=\frac{6}{\pi^{2}}
$$

and

$$
\prod_{p \mid m}\left(1-\frac{1}{p^{2}}\right)=\prod_{p \mid m}\left(1-\frac{1}{p}\right) \prod_{p \mid m}\left(1+\frac{1}{p}\right)=\frac{\varphi(m)}{m} \prod_{p \mid m}\left(1+\frac{1}{p}\right),
$$

we obtain

$$
\sum_{\substack{1 \leq d \leq \delta \\ \operatorname{gcd}(d, m)=1}} \frac{\mu(d)}{d^{2}}=\frac{6}{\pi^{2}} \frac{m}{\varphi(m)} \prod_{p \mid m}\left(1+\frac{1}{p}\right)^{-1}+O\left(\delta^{-1}\right) .
$$

We now substitute (9) and (10) in (8), which yields

$$
\begin{aligned}
N_{m}(U, V)= & \frac{6}{\pi^{2}} \cdot \frac{U V}{m} \prod_{p \mid m}\left(1+\frac{1}{p}\right)^{-1} \\
& +O\left(\delta m^{1 / 2+o(1)}+U V \delta^{-1} m^{-1}+\Delta m^{o(1)}+U V \Delta^{-1} m^{-1}\right) .
\end{aligned}
$$

Taking

$$
\delta=\left\lceil U^{1 / 2} V^{1 / 2} m^{-3 / 4}\right\rceil \text { and } \quad \Delta=\left\lceil U^{1 / 2} V^{1 / 2} m^{-1 / 2}\right\rceil,
$$

we derive the desired result.

It is easy to see that

$$
\prod_{p \mid m}\left(1+\frac{1}{p}\right) \ll \prod_{p \mid m}\left(1-\frac{1}{p}\right)^{-1}=\frac{m}{\varphi(m)} \ll \log \log m .
$$


In particular, we conclude that Theorem 3 is nontrivial under the condition (1).

COROLlary 4. For all integers $m$ and real $U, V$ with $1 \leq U, V<m$ and $U V \geq m^{3 / 2+\varepsilon}$, we have

$$
N_{m}(U, V)=\left(\frac{6}{\pi^{2}}+O\left(m^{-\varepsilon / 2+o(1)}\right)\right) \frac{U V}{m} \prod_{p \mid m}\left(1+\frac{1}{p}\right)^{-1} .
$$

4. Remarks. There is little doubt that our approach can also be used to obtain asymptotic formulas for the sums

$$
\sum_{(u, v) \in \mathcal{H}_{m}(U, V)}|\mu(u v)| \text { and } \sum_{(u, v) \in \mathcal{H}_{m}(U, V)}|\mu(u) \mu(v)|
$$

and several other sums. However, we do not see any approaches to bound the sums

$$
\sum_{(u, v) \in \mathcal{H}_{m}(U, V)} \mu(u v) \text { and } \sum_{(u, v) \in \mathcal{H}_{m}(U, V)}\left(\frac{u}{v}\right),
$$

where $(u / v)$ is the Jacobi symbol, which we also extend to even values of $v$ by putting $(u / v)=0$ if $\operatorname{gcd}(v, 2)=2$.

Various properties of points on multidimensional hyperbolas

$$
u_{1} \cdots u_{k} \equiv 1(\bmod m)
$$

have been studied as well $[1,21,22]$.

Hyperbolas $u v \equiv a(\bmod m)$ for an arbitrary integer $a$ with $\operatorname{gcd}(a, m)=1$ are also of interest. Although for every given $a$ their theory is similar to the case $a=1$, these new settings lead to a new type of problem of getting more precise results on average over $a$ (see $[6-10,16,19,23,31]$ and references therein)

Finally, solutions of more general polynomial congruences have also been studied in the literature (see for example [3, 4, 11, 25, 32]).

\section{References}

[1] E. Alkan, F. Stan and A. Zaharescu, Lehmer k-tuples, Proc. Amer. Math. Soc. 134 (2006), 2807-2815.

[2] F. P. Boca, C. Cobeli and A. Zaharescu, Distribution of lattice points visible from the origin, Comm. Math. Phys. 213 (2000), 433-470.

[3] C. Cobeli and A. Zaharescu, Generalization of a problem of Lehmer, Manuscripta Math. 104 (2001), 301-307.

[4] - - - On the distribution of the $\mathbb{F}_{p}$-points on an affine curve in $r$ dimensions, Acta Arith. 99 (2001), 321-329. 
[5] K. Ford, M. R. Khan, I. E. Shparlinski and C. L. Yankov, On the maximal difference between an element and its inverse in residue rings, Proc. Amer. Math. Soc. 133 (2005), 3463-3468.

[6] M. Z. Garaev, Character sums in short intervals and the multiplication table modulo a prime, Monatsh. Math. 148 (2006), 127-138.

[7] - On the logarithmic factor in error term estimates in certain additive congruence problems, Acta Arith. 124 (2006), 27-39.

[8] M. Z. Garaev and A. A. Karatsuba, On character sums and the exceptional set of a congruence problem, J. Number Theory 114 (2005), 182-192.

[9] —, - , The representation of residue classes by products of small integers, preprint, 2006.

[10] M. Z. Garaev and K.-L. Kueh, Distribution of special sequences modulo a large prime, Int. J. Math. Math. Sci. 50 (2003), 3189-3194.

[11] A. Granville, I. E. Shparlinski and A. Zaharescu, On the distribution of rational functions along a curve over $\mathbb{F}_{p}$ and residue races, J. Number Theory 112 (2005), 216-237.

[12] M. N. Huxley and W. G. Nowak, Primitive lattice points in convex planar domains, Acta Arith. 76 (1996), 271-283.

[13] H. Iwaniec and E. Kowalski, Analytic Number Theory, Amer. Math. Soc., Providence, RI, 2004.

[14] M. R. Khan and I. E. Shparlinski, On the maximal difference between an element and its inverse modulo n, Period. Math. Hungar. 47 (2003), 111-117.

[15] M. R. Khan, I. E. Shparlinski and C. L. Yankov, On the convex closure of the graph of modular inversions, preprint, 2006.

[16] H. N. Liu and W. Zhang, On a problem of D. H. Lehmer, Acta Math. Sin. (Engl. Ser.) 22 (2006), 61-68.

[17] H. L. Montgomery, Ten Lectures on the Interface between Analytic Number Theory and Harmonic Analysis, Amer. Math. Soc., Providence, RI, 1994.

[18] W. G. Nowak, Primitive lattice points inside an ellipse, Czechoslovak Math. J. 55 (2005), 519-530.

[19] I. A. Semaev, On the number of small solutions of a linear homogeneous congruence, Mat. Zametki 50 (1991), no. 4, 102-107 (in Russian).

[20] I. E. Shparlinski, On exponential sums with sparse polynomials and rational functions, J. Number Theory 60 (1996), 233-244.

[21] - , On the distribution of points on multidimensional modular hyperbolas, Proc. Japan Acad. Sci. Ser. A, to appear.

[22] - On a generalisation of a Lehmer problem, preprint, 2006.

[23] - Distribution of inverses and multiples of small integers and the Sato-Tate conjecture on average, preprint, 2006.

[24] G. Tenenbaum, Introduction to Analytic and Probabilistic Number Theory, Cambridge Univ. Press, 1995.

[25] M. Vajaitu and A. Zaharescu, Distribution of values of rational maps on the $\mathbb{F}_{p^{-}}$ points on an affine curve, Monatsh. Math. 136 (2002), 81-86.

[26] W. G. Zhai, On primitive lattice points in planar domains, Acta Arith. 109 (2003), $1-26$.

[27] W. P. Zhang, On a problem of D. H. Lehmer and its generalization, Compos. Math. 86 (1993), 307-316.

[28] —, On a problem of D. H. Lehmer and its generalization, II, ibid. 91 (1994), 47-56. 
[29] W. P. Zhang, On the difference between a D. H. Lehmer number and its inverse modulo q, Acta Arith. 68 (1994), 255-263.

[30] -, On the distribution of inverses modulo n, J. Number Theory 61 (1996), 301-310.

[31] —, On a problem of D. H. Lehmer and Kloosterman sums, Monatsh. Math. 139 (2003), 247-257.

[32] Z. Y. Zheng, The distribution of zeros of an irreducible curve over a finite field, J. Number Theory 59 (1996), 106-118.

Igor E. Shparlinski

Department of Computing

Macquarie University

Sydney, NSW 2109, Australia

E-mail: igor@ics.mq.edu.au

Received September 19, 2006;

received in final form November 21, 2006 